\title{
Diffuse reflectance measurement tool for laparoscopic surgery
}

\author{
Mario E. Giardini ${ }^{\mathrm{a}^{*}}$, Annett B. Klemm ${ }^{\mathrm{a}}$, Andrea Di Falco ${ }^{\mathrm{a}}$, Thomas F. Krauss ${ }^{\mathrm{a}}$ \\ ${ }^{a}$ SUPA - University of St Andrews - School of Physics and Astronomy - North Haugh - \\ St Andrews KY16 9SS - United Kingdom
}

\begin{abstract}
Continuous-wave diffuse reflectance or Near Infrared Spectroscopy (NIRS) offers the possibility to perform a preliminary screening of tissue for ischemia or other tissue anomalies. A tool for intracavity NIRS measurements during laparoscopic surgery, developed within the framework of the FP7-IP ARAKNES (Array of Robots Augmenting the KiNematics of Endoluminal Surgery) project, is described. It consists of a probe, that is located on the tip of an appropriately shaped laparoscopic manipulator and then applied to the tissue. Such a probe employs an array of incoherent semiconductor light sources (LEDs) frequency-multiplexed on a single detector using a lock-in technique. The resulting overall tool structure is simple and compact, and allows efficient coupling of the emitted light towards the tissue. The tool has high responsivity and enables fast and accurate measurements. A dataset gathered from in-vivo tissue is presented. The performance both indicates direct applicability of the tool to significant surgical issues (ischemia detection), and clearly indicates the possibility of further miniaturizing the probe head towards catheterized approaches.
\end{abstract}

Keywords: Laparoscopy, NIRS, robotic surgery, ARAKNES

\section{INTRODUCTION}

During surgery, the operator often needs to determine the tissue blood perfusion on a small portion of tissue. Possibly the most notable example for this requirement is immediately after applying stitches (suturing), when underperfusion of the tissue affected by the procedure indicates a potentially flawed suture, that will fail after a short time.

Such determination, essential during most surgical procedures, is normally performed by visual inspection. However, during laparoscopic surgery which takes place inside a dilated body cavity, the tissue is not visualized directly. Rather, illumination sources need to be coupled to the cavity in which the surgery is being performed, and the process is prone to color temperature control issues. The tissue is then visualized using complex optical instruments or video imaging systems that need to perform a color rendering. As the feedback used by the surgeon to evaluate perfusion ultimately relies on fine hints related to tissue color, direct laparoscopic viewing exposes perfusion determination to artifacts due to color distortion.

The situation is further aggravated in robotic surgery, where the operator does not gain direct access to the laparoscopic cavity. Surgical robots, and the associated robotic tools, are driven by one or more remote consoles, and the necessity to optimize the remote viewing system for three-dimensional surgical volume rendition, often does not account for the continuous shifting and optimization of the viewing point that is normally performed during manually-assisted surgery to, at least partially, compensate for the highly sub-optimal view of the operating field intrinsic to laparoscopy.

Assistive surgical laparoscopic tools for the determination of tissue oxygenation that could, at least partially, relieve the surgeon from the task of determining tissue perfusion by observation of the color only, would therefore, be particularly attractive.

In principle, the problem of determining tissue perfusion can be shifted from direct visual observation to spectroscopic optical analysis of the tissue. From an optical point of view, biological tissue can be considered as an absorbing matrix in which a high number of inhomogeneities, which act as light scatterers, are present ${ }^{1}$. When collimated near-infrared (NIR) light is injected into the tissue and the collimated transmission is observed, collimated light losses are mostly due to scattering (i.e., to light deviation) rather than to absorption. This allows light to travel around the tissue

\footnotetext{
**Correspondence: mario.giardini@st-andrews.ac.uk - Tel. +44 (0)1334 463439 - Fax +44 (0)1334 463104
}

Biophotonics: Photonic Solutions for Better Health Care II, edited by Jürgen Popp,

Wolfgang Drexler, Valery V. Tuchin, Dennis L. Matthews, Proc. of SPIE Vol. 7715, 77150Z

(C) 2010 SPIE · CCC code: 1605-7422/10/\$18 - doi: 10.1117/12.854127 
and, eventually, emerge from the light injection surface. From the analysis of the backscattered light ("diffuse reflectance") and, in essence, from the comparison between the light expected in absence of absorption and of the intensity actually measured, the amount of tissue absorption, and with it, the concentration of light-absorbing substances, can be determined.

In the NIR range, the optical absorption is dominated by haemoglobin, the blood constituent that carries oxygen by fixing it in an oxygenated form (oxyhaemoglobin), subsequently releasing it into the tissue, thus reverting to a deoxygenated form (deoxyhaemoglobin). The absorption spectra of haemoglobin ${ }^{2}$ are reported in Fig. 1. We note that below $800 \mathrm{~nm}$ (isosbestic point) the absorption is strongly dominated by deoxyhaemoglobin, while above the isosbestic point oxyhaemoglobin absorption prevails. A small contribution to absorption is given by cytochrome aa3, another molecule involved in oxygen metabolism. Again, such molecule has an oxidized and a reduced form; a quantitative determination of the differential absorption spectrum may give information on tissue oxygen consumption. However, the correction term introduced by cytochrome aa3 accounts for a small percentage of the absorption only ${ }^{3}$. The last NIR absorption source, which may be especially present in transdermal measurements, is epithelial pigmentation; however, such absorption term, if any, does not change with oxygen saturation.

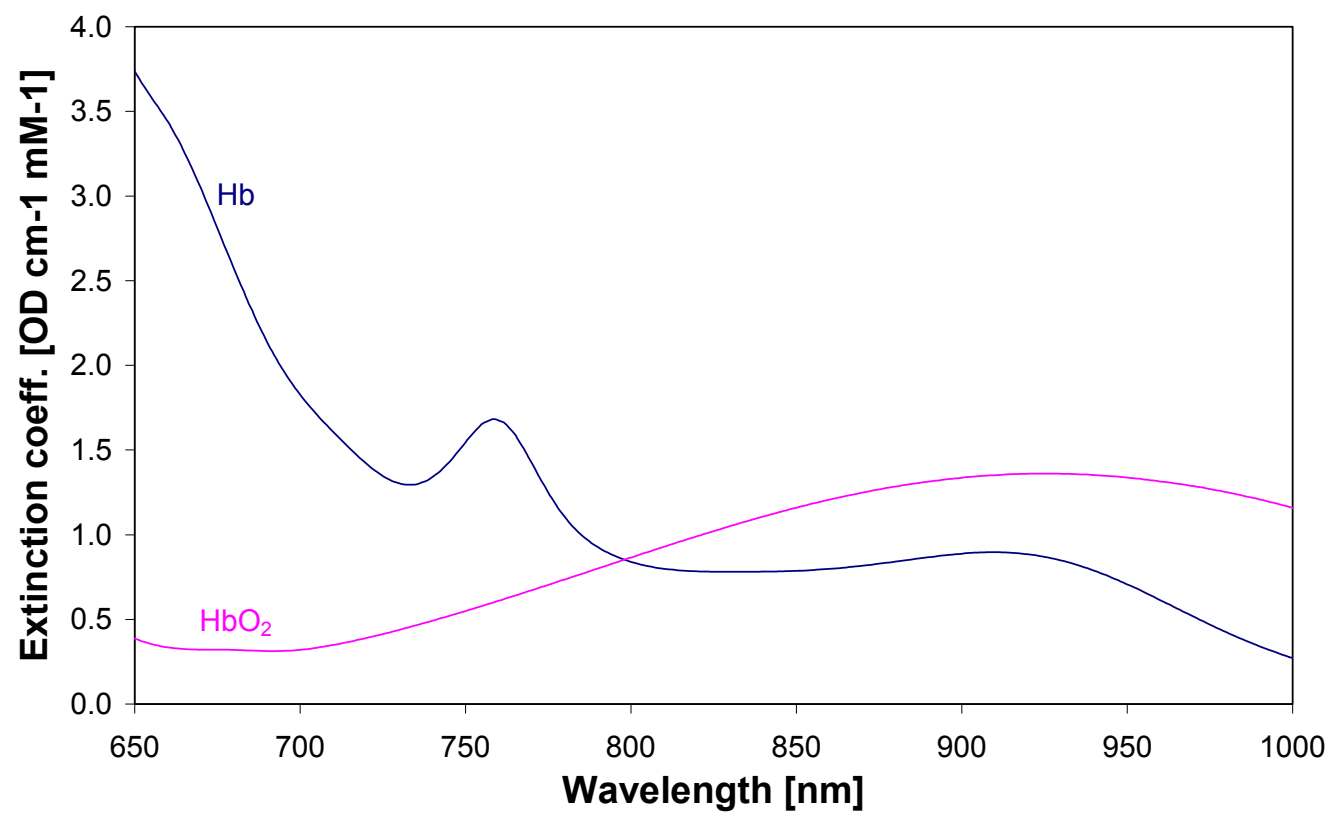

Fig. 1 - NIR extinction coefficient for oxyhaemoglobin $\left(\mathrm{HbO}_{2}\right)$ and deoxyhaemoglobin $(\mathrm{Hb})$, from ref. 2.

While certainly simple from a theoretical and instrumental point of view, in-vivo determination of blood oxygenation by optical transmission requires a sampled volume, which should be transmissive enough to detect the transmitted light. Mainly because of scattering losses, this limits the application of direct transmissive measurements to small tissue thicknesses. In the last twenty years, a number of instruments have therefore been constructed, which determine tissue oxygenation by using optical spectrum analysis of the light backscattered by a depth of a few millimeters under the surface of thick tissue (Near InfraRed Spectroscopy - NIRS, or diffuse reflectance analysis) ${ }^{4,5}$.

Mainly because of the difficulties in the clinical interpretation of the resulting signals ${ }^{3}$, the field is still open to clinical and instrumental research. In particular, to our knowledge, the applicability of infrared backscattering for ischemia detection, key issue in robotic surgery, is yet completely to be explored. In this paper, a tool for diffuse reflectance (NIRS) measurements in endoscopic and intracavitary surgery is presented. In constituting a useful tool per $s e$, it is built as a pilot study, in view of further and better optimized robotic implementations, within the framework of the ARAKNES surgical robotic platform ${ }^{6}$. 


\section{THE TOOL}

The tool consists of a laparoscopic probe connected to a preamplifier. Our probe, reported in Fig. 2., is designed towards single-port laparoscopic surgery, such as allowed by transumbilical insertion devices ${ }^{7}$. As required by such technique, it is constituted by a straight hollow shaft, $330 \mathrm{~mm}$ long, with $4 \mathrm{~mm}$ outer diameter and $3.5 \mathrm{~mm}$ inner diameter $(5.1 \mathrm{~mm}$ being the highest standard diameter allowed without occlusion of the main laparoscopic access, which should remain open for higher-priority tools).

Such shaft is connected to a cylindrical handle, $200 \mathrm{~mm}$ long and $20 \mathrm{~mm}$ in diameter. For the prototypal nature of the tool, the shaft and handle are in-line and coaxial and this, upon insertion, calls for extraction of eventual other inline surgical tools to avoid handle interference on the umbilical access port.

Light at 660 and $850 \mathrm{~nm}$ peak wavelengths is emitted by two epoxy-encapsulated LEDs (Kingbright L-535RCE and Osram SFH4550 respectively). Cylindrical holes have been drilled in the epoxy encapsulation towards the LED chips, and $1 \mathrm{~mm}$ core polymeric optical fibers have been inserted in the LEDs and glued in the holes with cyanoacrylic glue, transparent in the full wavelength range of interest., thus proximity-coupling the emitting chips and the fibers. Similarly, a $1 \mathrm{~mm}^{2}$ epoxy-encapsulated front-looking silicon photodiode (Osram SFH213) has been drilled and glued to an analogous optical fiber.

The two emitting fibers have been inserted into the shaft, and glued to the inside surface of the shaft tip, both on one side. The detection fiber has been glued, still on the inside surface of the shaft tip, on the opposite side of the emitting fibers, thus resulting in a $3 \mathrm{~mm}$ separation between emitting and receiving fibers. The LEDs and the photodiode have been located in the handle, and connected to a LED driver/photodiode amplifier by two separate multicore shielded cables $2 \mathrm{~m}$ long.

The shaft and the distal $180 \mathrm{~mm}$ of the handle have been internally filled with an optically shielding epoxy potting compound, and the remaining proximal $20 \mathrm{~mm}$ have been sealed with an elastic silicone resin, that acts as curvature stress relief ferrule for the cables. The resins, the fibers and the shaft material (brass), though sterilizable by cold techniques, such as exposure to ethylene oxide or to surgical sanitizers, are currently not qualified for intrasurgical in-vivo use. However, for the specific purpose of pilot acute animal studies, the materials have been accepted by our collaborating surgical teams.

In such configuration, the sensing surface of the tool is located into the tip of the shaft, which has been cut and polished to a flat bevel, tilted 20 degrees with respect to the shaft axis. This geometry allows the positioning of the tip flat on the tissue to be examined, with no pressure "hot spots" and with maximal maneuverability in tip placement.

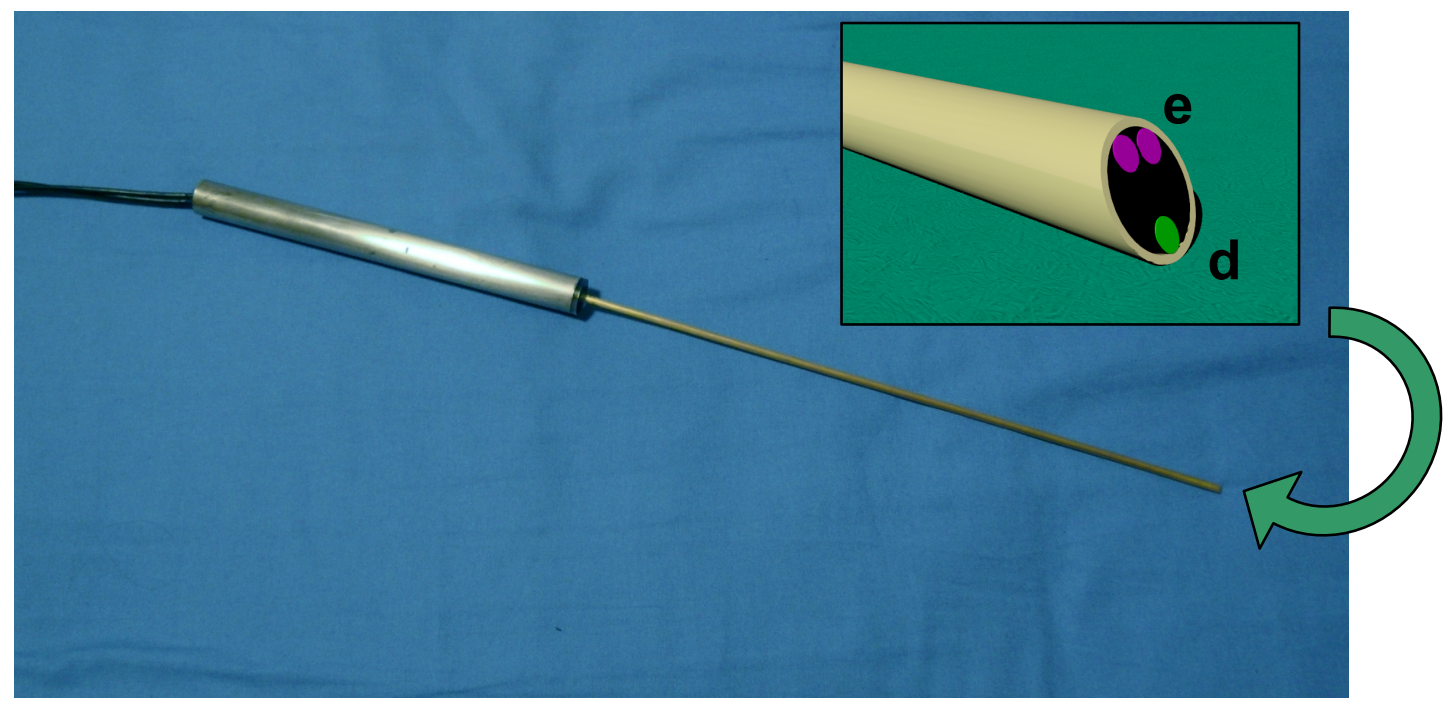

Fig. 2 - The laparoscopic probe. In the insert, a rendering of the tip, showing the emission (e) and detection (d) fibers 
Light is emitted by the LEDs, reaches the tip through the emitting fibers, propagates into the tissue, and the backscattered light is guided by the sensing fibers to the photodiode. In order to separate the backscattering contribution from the individual sources, the LEDs are modulated at two different frequencies (nominally $1.1 \mathrm{and} 1.2 \mathrm{kHz}$ ) and the signal from the photodetector is demodulated synchronously at the two frequencies, yielding the two independent signals, as described elsewhere ${ }^{8}$.

\section{PRELIMINARY PERFORMANCE TEST}

All literature protocols for the determination of oxygen saturation ${ }^{3}$ rely on the knowledge a priori of the intensities of the light emitted into the tissue by the different sources. In order to measure such intensities, we applied the probe to a non-absorbing tissue phantom prepared, according to ref. 9, by dispersing aluminum oxide particles in a silicone matrix. The reduced scattering coefficient ${ }^{10}\left(\mu_{\mathrm{s}}^{\prime}\right)$ of our phantom has been measured ${ }^{11}$ to be $0.261 \pm 0.006$, representative of an actual in-vivo muscle tissue.

As the tool is designed to yield saturation values only, and no total haemoglobin concentration is needed, we require the relative source intensities only. The increase, in the phantom, of the optical pathlength with respect to the tissue due to the lack of optical absorption is therefore inessential, and it has been neglected.

The in-vivo performance of the tool has been tested on a benchmark protocol known as tourniquet-induced forearm ischaemia ${ }^{12}$. Such test is represented in Fig. 3. A patient is at rest, in a stationary state with respect to blood pressure, heart rate and muscle fatigue. An inflatable cuff (tourniquet) is applied around the patient arm and inflated in order to shut off the blood supply to the forearm. During the ischaemia, oxygen consumption in the forearm is not interrupted, and thus the blood in the forearm is progressively deoxygenated. The forearm vessels respond to such deoxygenation by trying to increase the overall perfusion, hindered into this by the mechanical constraint that total blood volume is kept constant by the occlusion. The tourniquet is then released, blood starts flowing again through the arm and oxygenation recovers its initial value, Since all volume constraints are removed, the vessels can increase their size, and so blood volume and oxygenation typically overshoot the stationary value, which is normally recovered several minutes after ischaemia removal ${ }^{13}$.

The protocol has been performed on a male 42-year old volunteer. The probe has been applied by lightly touching with the shaft tip the skin over the flexor segments of the forearm muscles, a few seconds at a time, before and during the ischemization, that has been maintained for 3 minutes. As the probe presents a $3 \mathrm{~mm}$ source-detector separation, we can expect the measurement to be scarcely representative of the muscle tissue, as the measurement depth is insufficient to reach into the muscle body, and only the skin, which nevertheless undergoes ischemia, is explored.

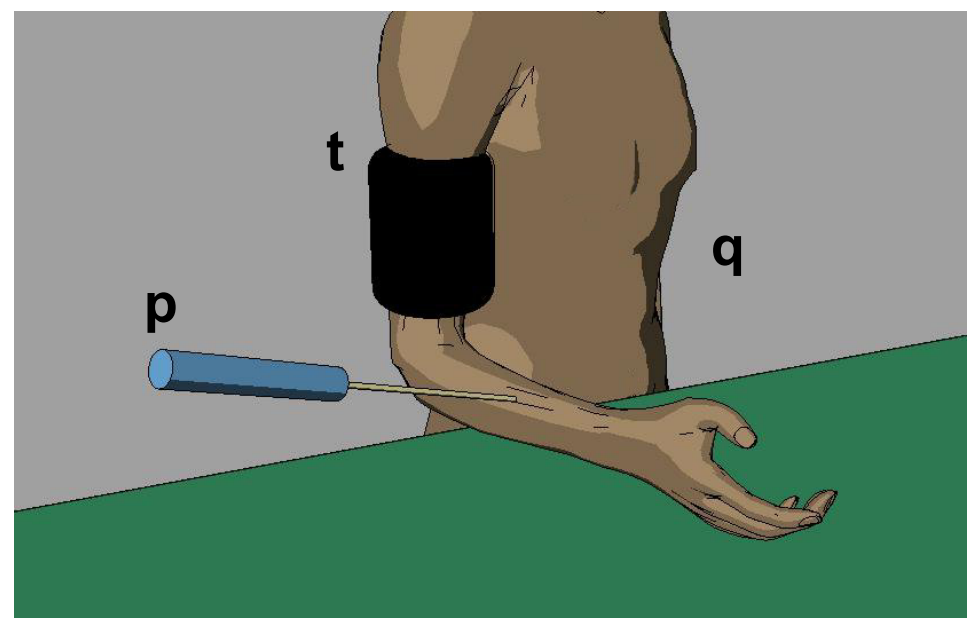

Fig. 3 - Tourniquet-induced forearm ischaemia: (p) probe, (t) tourniquet, (q) patient. 
The saturation data has then been determined by direct application of the Lambert-Beer law ${ }^{1}$, whose accuracy we have deemed sufficient to contrast between healthy and ischemized tissue, such as encountered in surgery. The resulting data is reported in Fig. 4.

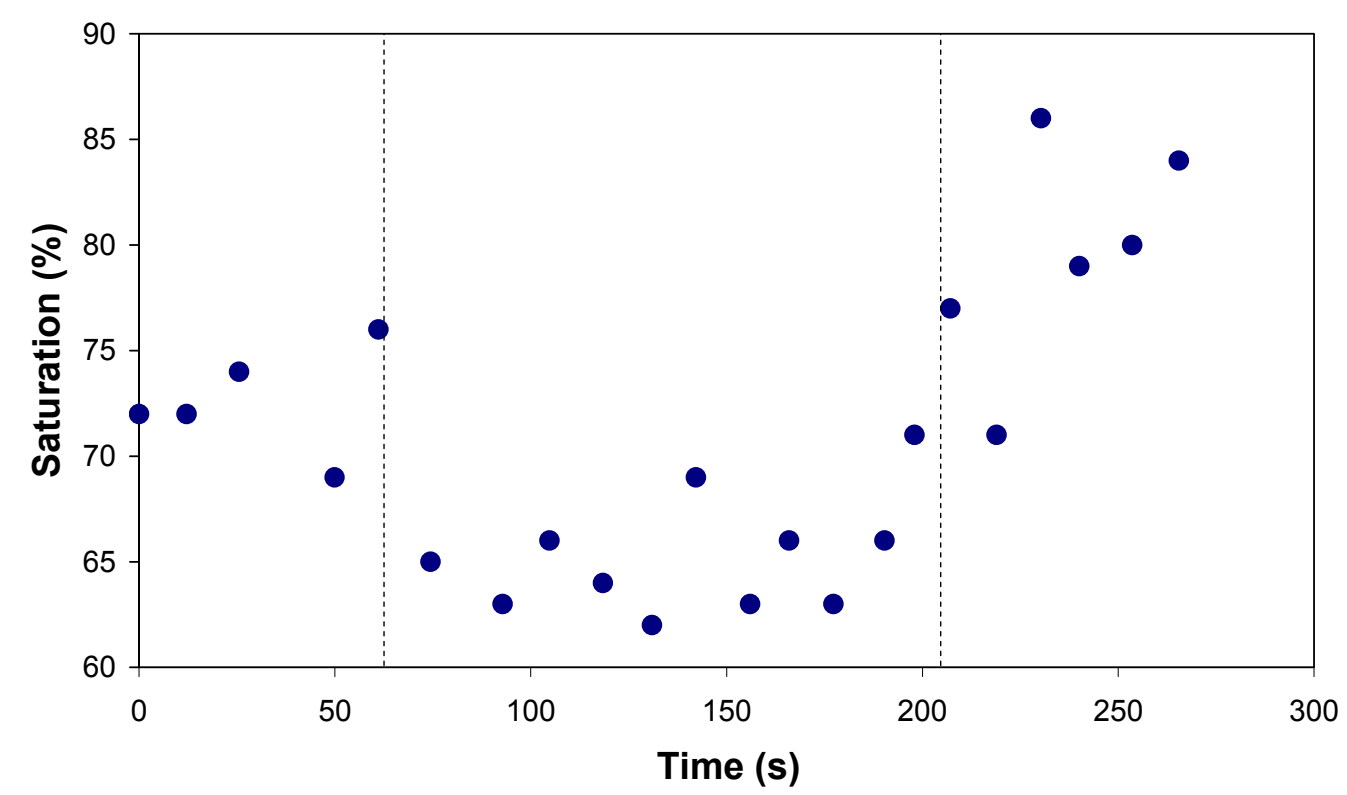

Fig. 4 - Saturation as measured on the forearm by the tool during tourniquet-induced forearm ischaemia. The dotted lines indicate ischaemia onset and release.

We note a visible quick drop in saturation at the onset of the occlusion, that is rapidly recovered as the occlusion is released, overshooting the intial values. The relatively low contrast upon occlusion (from $70-75 \%$ to 60 $65 \%$ ) is typical ${ }^{12}$, and it is dictated by the need to keep discomfort to the volunteer to a minimum. In intrasurgical conditions, the contrast between healthy and ischemized tissue is expected to be much greater as, in clinically relevant cases, blood supply is typically completely occluded for periods longer than the 3-minute tourniquet test, and as no significant skin layers, that are less homogeneous than bulk tissue, are present.

In particular, signal fluctuations are known to arise from probe application site variability ${ }^{14}$. The signal stability would therefore greatly benefit from the possibility to fix the probe location for the duration of the surgical procedure. To this respect, the mere visibility of a contrast between perfused and ischemized tissue even in such unfavorable conditions as transdermal measurement of tourniquet-induced ischemia, is strongly suggestive of the actual possibility to improve the probe performance by further miniaturization, so as to allow to deliver it on the measurement site through a cathererised approach, and to leave it in situ for the required amount of time.

\section{CONCLUSIONS}

We have shown a tool for diffuse reflectance measurements during laparoscopic surgery. A preliminary in-vivo test on tourniquet-induced forearm ischaemia shows a response to tissue ischemization. The actual sensitivity during surgical conditions still needs to be explored, though it can be expected to improve with respect to the current extrasurgical setting, and thus be promising for future robotic surgery implementations. 


\section{ACKNOWLEDGMENTS}

We wish to acknowledge funding from the European Union under Agreement no. 224565 "ARAKNES: Array of Robots Augmenting the KiNematics of Endolunimal Surgery". We thank Sir Alfred Cuschieri and Dr Andrea Peri, part of the ARAKNES surgical team, for invaluable discussion on the surgical ergonomics.

Intellectual and exploitation rights related to some technologies outlined in the present paper are owned by the University of St Andrews (UK), that should be contacted for any further information.

[1] Cheong, W., Prahl, S. A. and Welch, A. J., "A Review of the Optical Properties of Biological Tissues", IEEE J. Quantum Elec. 26, 2166-2185 (1990).

[2] Cope, M., Ph.D. thesis, University College London, U.K. (1991).

[3] Matcher, S. J., Elwell, C. E., Cooper, C. E. and Delpy, D. T., "Performance Comparison of Several Published Tissue Near-Infrared Spectroscopy Algorithms", Anal. Biochem. 227, 54-68, (1995).

[4] Owen-Reece, H., Smith, M., Elwell, C. E. and Goldstone, J. C., "Near Infrared Spectroscopy”, Br. J. Anaesth. 82, 418-426 (1999).

[5] Wahr, J. A., Tremper, K. K., Samra, S. and Delpy, D. T., "Near-Infrared Spectroscopy: Theory and Applications", J. Cardiothor. Vasc. Anaesth. 10, 406-418 (1996).

[6] European Union - Framework Programme 7 - Project no. 224565 "ARAKNES: Array of Robots Augmenting the KiNematics of Endolunimal Surgery", www.araknes.org

[7] Desai, M. M., Rao, P. P., Aron, M., Haber, G-P, Desai, M. R., Mishra, S., Kaouk, J. H. and Gill, I. S., "Scarless single port transumbilical neprectomy and pyeloplasty: first clinical report", BJU International 101, 83-88 (2008).

[8] Giardini, M. E., Corti, M., Lago, P. and Gelmetti, A., "Portable Microcontroller-Based Instrument for Near Infrared Spectroscopy", Proc. SPIE 3911, 250-255 (2000).

[9] Bays, R., Wagnières, G., Robert, D., Theumann, T., Vitkin, A. Savary, J., Monnier P. and van den Bergh, H., "Threedimensional optical phantom and its application in photodynamic therapy", Las. Surg. Med. 21, 227-234 (1997).

[10] Tuchin, V., [Tissue Optics], SPIE Publishing, USA (2000).

[11] Giardini, M. E., Loconte, L., Guizzetti, G., Gelmetti, A. and Lago, P., "Simple Instrument for the Characterization of Diffuse Reflectance", Proc. SPIE 3568, 59-63 (1999).

[12] Hampson, N. B. and Piantadosi, C. A., "Near infrared monitoring of human skeletal muscle oxygenation during forearm ischemia", J. Appl. Physiol. 64, 2449-2457 (1988).

[13] Giardini, M. E., Guizzetti, G. G., Bavera, M., Lago, P., Corti, M. and Falcone, C., "Near-infrared Spectroscopy Study of Tourniquet-Induced Forearm Ischaemia in Patients with Coronary Artery Disease", Proc. SPIE 4254, 1-6 (2001).

[14] Bianchi, T., Zambarbieri, D., Beltrami, G. and Verni, G., "NIRS monitoring of muscle contraction to control a prosthetic device", Proc. SPIE 3570, 157-163 (1999). 